Sociologie et sociétés

\title{
La communication en France socialiste
}

Des difficultés de conjuger technologie et démocratie

Communications in Socialist France

Difficulties in Bringing Together Technology and Democracy

\section{Armand MATTELART}

Volume 16, numéro 1, avril 1984

L’informatisation : mutation technique, changement de société?

URI : https://id.erudit.org/iderudit/001482ar

DOI : https://doi.org/10.7202/001482ar

Aller au sommaire du numéro

\section{Éditeur(s)}

Les Presses de l'Université de Montréal

\section{ISSN}

0038-030X (imprimé)

1492-1375 (numérique)

Découvrir la revue

Citer cet article

MATTELART, A. (1984). La communication en France socialiste : des difficultés de conjuger technologie et démocratie. Sociologie et sociétés, 16(1), 125-144. https://doi.org/10.7202/001482ar 


\section{La communication en France socialiste: Des difficultés de conjuguer technologie et démocratie}

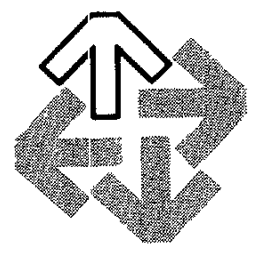

ARMAND MATTELART

\section{DES AXES D'ACTION CONFLICTUELS}

Sous des angles divers, à un titre ou à un autre, comme simples vœux pieux ou comme signe de nouvelles réalités émergentes, le thème de la démocratie et de son rapport avec les nouvelles technologies de communication et d'information a depuis la victoire socialiste de mai 1981 été au centre de nombreux discours, propositions et projets, officiels et officieux. «Démocratiser l'informatique» au lieu «d'informatiser la société»: c'est ce que déclarait le président Mitterand au salon de l'informatique (SICOB) à l'automne 1981 prenant ainsi le contre-pied de la politique du septennat précéclent. Pour l'extension des nouveaux réseaux ou des nouveaux services de communication, on n'obéirait plus au déterminisme de l'offre technologique; on n' «imposerait» plus l'annuaire électronique à tout le monde, on le «proposerait»; on tenterait de laisser aux usagers les choix télématiques; on garantirait aux salariés que l'introduction des nouvelles technologies dans leur travail se ferait avec leur concours. Bref, on redécouvrait qu' «une technologie, pour se développer, doit bénéficier d'un environnement social favorable ${ }^{1}$ ».

Plus substantiellement, le colloque national de la recherche et de la technologie, large consultation de la communauté scientifique qui a précédé l'élaboration de la loi d'orientation et de programmation pour la recherche et le développement technologique de la France, posait l'urgence de «restaurer l'alliance de la science et de la démocratie ${ }^{2}$ ». Entre novembre 1981 et fin janvier: 1982, trente et une assises régionales préparaient à travers tout le pays les journées nationales de ce colloque ancré dans l'idée que «consulter avant d'agir n'est pas seulement une démarche propre à la méthode scientifique mais aussi, rejoignant celle-ci, à l'esprit de la démocratie... ${ }^{3}$ \%. Furent élaborées des milliers de contributions par les organismes de recherche, les universités, les entreprises, les syndicats, les organisations professionnelles, créant de la sorte un mouvement de réflexion sans précédent dans l'histoire de la recherche française, tentant de susciter une vaste prise de conscience tant de la part des artisans mêmes de la science et de la technologie que de leurs utilisateurs actuels ou potentiels. En décidant de toucher tous les partenaires des choix technologiques

1. J.P. Chevènement, ministre de la Recherche et de la Technologie, dans le Monde, 23 septembre 1981, p. 1.

2. Ministère de la Recherche et de la Technologie, Recherche et technologie, Actes du colloque national 13-16 janvier 1982, Paris, La Documentation Française, 1982, Préface, p. 7.

3. Ibid. 
à effectuer, le colloque tenta de faire de la recherche et des avancées technologiques «un enjeu national dans lequel chacun se sent concerné».

Les relations entre la science, la technologie et les autres grands domaines de l'activité sociale - lit-on dans le document préparatoire au colloque - ne peuvent rester du ressort des seuls spécialistes qui assument la responsabilité de leur développement. Il est vrai que les scientifiques sont les mieux placés pour savoir quelles sont les directions de recherche les plus prometteuses, et il est non moins vrai que c'est dans le champ industriel et commercial qu'on perçoit le mieux les perspectives d'application du savoir. Mais le dialogue entre les spécialistes et les non-spécialistes ne pourra se nouer que sous deux conditions. La première est de rendre transparentes les controverses qui se développent à l'intérieur des sciences et des techniques. C'est en effet là qu'apparaissent et se discutent les choix possibles; c'est là qu'il faut établir d'abord et de manière durable une première forme d'expression démocratique: celle des idées et des projets scientifiques et techniques. La deuxième condition est de ne laisser ni aux scientifiques ni aux ingénieurs l'exclusivité des décisions majeures susceptibles d'engager l'avenir du pays. Le Gouvernement, le Parlement, les instances régionales, les syndicats, les associations - la liste n'étant pas limitative - doivent, chacun selon ses responsabilités, pouvoir accéder aux connaissances nécessaires pour être en mesure de prendre parti de façon éclairée. En fait, c'est la communauté nationale tout entière qui, au travers de ses représentants qualifiés, doit pouvoir «contrôler» les choix à partir d'une information complète des citoyens sur toutes leurs implications ${ }^{4}$.

Disons-le d'entrée de jeu. Cette perspective qui définit le rapport démocratie-technologie en termes d'appropriation par la société dans son ensemble des nouveaux outils technologiques n'a pas toujours été présente dans les discussions ou projets qui, en d'autres lieux institutionnels, ont eu pour objet les nouveaux outils d'information et de communication. C'est une problématique par exemple qui est loin d'être au centre des préoccupations du rapport de la commission de réflexion et d'orientation sur l'audiovisuel (rapport Moinot) rendu public à la mi-octobre $1981^{5}$. Même si des propositions sont faites dans le sens de la décentralisation, même si affleurent de timides références à la démocratisation de l'accès à l'antenne, la conception générale qui se dégage du rapport est celle d'un droit à communiquer construit trop unilatéralement sur le droit inaliénable des professionnels à communiquer, à bien faire leur métier. L'idéologie professionnaliste semble avoir fixé les limites dans lesquelles il est possible de discuter de la «démocratisation» de l'information.

C'est dire que les grands axes qui ont traversé le débat sur la recherche - la nécessité de repenser la science et la technologie en fonction d'un nouveau schéma de relations entre partenaires sociaux - n'occupent guère de place dans ce premier rapport sur l'audiovisuel. Le droit de communiquer défini comme droit à être informé a largement prévalu sur le droit, qui devrait être tout aussi inaliénable, à produire sa propre information. On ne trouve guère dans ce rapport officiel les traces d'une réflexion sur les expériences multiples de vidéo et de cinéma, qui, en dehors des grands circuits commerciaux et du monopole de la radio-télévision ont, dans un mouvement de va et vient souvent contradictoire, au cours des quinze dernières années, avancé d'autres façons de définir le rapport communication-démocratie. On ne repère pas dans ce rapport ce qui serait l'équivalent de ce «droit à la recherche sociale» postulé par certains secteurs - malheureusement pas tous - lors du colloque national. Droit qui déniant aux milieux professionnels spécialisés la recherche comme leur apanage exclusif reconnaîtrait aux différents groupes sociaux la possibilité de devenir les promoteurs d'une recherche sur leur environnement social et matériel, sur les inégalités sociales et de décider eux-mêmes s'ils souhaitent ou non s'adjoindre les services de chercheurs professionnels ${ }^{6}$.

Les débats parlementaires sur la nouvelle loi de l'audiovisuel qui se sont déroulés en maijuin 1982 n'ont pas réussi à tordre le cou à cette logique corporatiste. Les vraies questions ont été éludées au seul bénéfice d'une discussion essentiellement juridique et institutionnelle, dont on ne peut certes nier la nécessité, mais qui n'est pas allée à l'essentiel. En privilégiant les interventions sur l'indépendance de l'information, à droite comme à gauche, on en a été réduit à ne voir le

4. «Annexe I: «Note d'orientation», ibid, p. 41.

5. Publié à la Documentation française, 1982.

6. Voir F. Guattari, «Note concernant un projet de fondation pour les initiatives locales, les innovations institutionnelles, la recherche en science sociale, l'animation et la création culturelles», Paris, février 1982, manuscrit. Dans le même sens d'une prise en compte des collectivités locales, associations, syndicats dans la «recherche - action», voir l'entretien avec Philippe Barret, responsable des sciences sociales au ministère de la Recherche et de la Technologie, «Les sciences de l'homme et de la société», Revue Non! (Repères pour le socialisme), Paris, février-mars 1982, $\mathbf{n}^{\circ} 12$, pp. 114-122. 
problème de la communication et de la démocratie que comme un enjeu politique à court terme et on a oublié l'enjeu culturel, c'est-à-dire les incidences d'un modèle de communication sur les formes mêmes de société. À nouveau, une certaine idée de l'indépendance des professionnels a laissé les parlementaires du pouvoir socialiste sur la défensive et a permis à une opposition amnésique de désigner et d'imposer son terrain comme lieu unique de discussion. En s'abritant sous le parapluie de la liberté (celle du journaliste), on cédait sur l'égalité. À force de n'insister que sur la nécessité de préserver l'indépendance des professionnels à l'égard du pouvoir politique, on a fini par rendre crédible l'idée de leur indépendance à l'égard des rapports sociaux. Tout se passe comme si on croyait qu'il suffit de faire disparaître les pressions du pouvoir politique sur les professionnels des médias pour que l'information et la pratique journalistique deviennent transparentes. Du même coup, toutes les autres questions devenaient superfétatoires: celle, par exemple, fondamentale, de déterminer comment à travers des pratiques sociales professionnellement codifiées dans un mode particulier de produire l'information se reproduisent un rapport à la réalité, un rapport à l'ensemble du corps social, bref une conception particulière d'ordonnancement du monde de chacun et de tous? ${ }^{7}$. Plus grave, à notre sens: en isolant la discussion sur la communication dans la sphère corporatiste ou de l'idéologie du journalisme, en revalidant le postulat de l'évidence, de la transparence du serıs, on faisait non seulement avancer l'idée de la neutralité de la «technique journalistique» mais on légitimait également une approche des technologies de communication qui allait dans le même sens technocratique.

Ces notes d'introduction ne sont pas là pour nous perdre en conjectures sur l'issue des politiques, formelles ou informelles, de communication en cuvre dans la réalité française d'aujourd'hui. Tant il est vrai qu'à force de jouer les Cassandre, on est souvent proche de la résignation. Ces notes ne sont là que pour laisser entrevoir le statut conflictuel du rapport communication-démocratie. Ce statut conflictuel trace une ligne d'horizon qu'il est difficile de laisser hors champ si l'on veut, à partir des divers mouvements de réalité qui opèrent dans la communication et l'information, faire émerger les axes d'une réflexion théorique sur le thème recherche - communication - démocratie. Pour cela, il faut évidemment accepter de secouer de nombreuses idées reçues sur ce qui constitue le champ d'observation propre à la communication comme discipline scientifique. Ce n'est pas le moindre problème. Qu'on le veuille ou pas, la polyvalence des nouveaux réseaux d'information et de communication impose non seulement la nécessité d'approches interdisciplinaires mais celle d'abordages intersectoriels des problèmes.

\section{DÉMOCRATIE ET STRATÉGIE INDUSTRIELLE}

Sans vouloir glisser dans l'économisme pour échapper au culturalisme des prophètes de la nouvelle agora électronique ou de la démocratie sur console qui ne se soucient guère de démocratie tout court, il faut quand même rappeler qu'une condition nécessaire (mais certainement pas suffisante) de l'exercice effectif de la démocratie est l'état de son économie. C'est-à-dire la façon dont une nation gère son patrimoine de production matérielle et symbolique, cette longue accumulation du vécu et de luttes des individus, des groupes et des classes; la façon dont cette communauté nationale, traversée de projets sociaux antagoniques, tente au besoin de récupérer ce patrimoine, de le construire ou de le reconstruire; la façon dont cette communauté met en œuvre, dans un projet de société, la créativité, l'innovation, l'inventivité des divers groupes sociaux qui la composent.

Pour définir davantage ce qu'est un «projet national», il faudrait s'aventurer dans des domaines pratiquement inexplorés par la recherche comme celui de la culture nationale et de son rapport dialectique avec le processus de transnationalisation. En ces temps où partout dans le monde la culture et l'identité nationale tendent à se transformer en un des axes des discours d'escorte des projets les plus opposés, il serait important de resituer ce concept dans le contexte concret des rapports de force dans lequel se construit et se reconstruit cette culture nationale. Il est quand même dommage de constater que la plupart du temps le «nationalisme», l'indépendance nationale ne deviennent des références opérationnelles que lorsqu'il s'agit de galvaniser un peuple contre un ennemi, un agresseur (commercial ou militaire). Mais c'est aussi à ce moment là que ces notions devenus symboles de rassemblement par de là les groupes et les classes perdent leur conflictualité. Ce n'est évidemment pas notre propos ici d'aller plus loin dans ce qui devrait devenir un champ important de la recherche critique.

7. Voir sur ce point A. Mattelart, Mass media, idéologies et mouvement révolutionnaire, Paris, Anthropos, 1974. 
Tout ceci pour dire qu'il est difficile si on veut parler de démocratie de ne pas faire un détour en se penchant sur la façon dont un pays mobilise les énergies autour d'une stratégie industrielle de reconquête de son indépendance technologique. Avec le changement politique du 10 mai et les nationalisations qui en ont découlé, cette stratégie occupe le devant de la scène. Est-il possible pour la France, et avec elle l'Europe, de maintenir une certaine autonomie face aux groupes américains et japonais? Évoquant la compétition internationale pour la maitrise des connaissances scientifiques et technologiques, le groupe de travail chargé de la préparation du colloque sur la recherche renvoyait à un discours du candidat présidentiel François Mitterand sur les grandes lignes de la politique de la recherche et de la technologie qu'il comptait mettre en ouvre après son élection:

La question posée est dès lors simple: quelle place pour la France dans cette compétition internationale? Â la tête ou à la traîne? Au cinquième rang, après les USA, l'URSS, le Japon, la RFA ou au premier rang? Vassale ou éclaireur? Mon choix est fait. Je veux qu'au cours du septennat prochain la France de la science soit à la tête du peloton ${ }^{8}$.

Sans nous demander maintenant quel écho rencontreraient chez un scientifique brésilien, chinois ou indien ces déclarations qui réagence le monde à partir d'une vision occidentale, contentonsnous de remarquer que la stratégie industrielle de la France mise sur les potentialités des technologies pour venir à bout de la crise. A ce titre, biotechnologies, électronique, nouvelles technologies de communication, nouveaux moyens énergétiques sont assumés comme des moyens de sortie de crise, des moyens qui devraient permettre une voie de développement concerté de l'économie mondiale, à condition d'en avoir la volonté politique. Une crise dont on reconnaît qu'elle a pour enjeux la redéfinition des rapports économiques et politiques entre les différentes nations, la redistribution des rapports de force tant internationaux qu'internes. Dans son rapport sur Technologie, emploi, croissance, présenté au Sommet de Versailles en juin 1982, le Président français affirmait:

Où en est aujourd'hui le progrès technique et que peut-il changer, dans les dix ans qui viennent, à la crise que nous traversons? Non seulement nous avons le devoir d'examiner ensemble, pour les résoudre, les problèmes posés par la crise et, pour cela, de s'accorder sur sa nature et sur ses causes, mais encore il reste à explorer de vastes champs ouverts à nos efforts communs. Parmi eux, celui qu'offrent la science et la technologie, dont l'évolution rapide entraîne le bouleversement de nos sociétés et risque de se retourner contre l'homme lui-même dont pourtant elles procèdent si celui-ci n'en assure pas la maîtrise 9 .

Même leitmotiv lors du colloque national sur la recherche:

Miser sur la recherche scientifique et le progrès technologique comme éléments moteurs pour sortir de la crise, ce n'est pas seulement les utiliser pour en combattre les effets directs, c'est aussi leur assigner une mission de grande envergure: celle de fournir les bases d'un autre modèle de développement de la société, susceptible de prendre le relais des modèles actuels en faisant de la connaissance et de son utilisation rationnelle les instruments privilégiés du renouveau ${ }^{10}$.

On peut très bien ne pas s'accommoder de cette vision des choses et mettre en avant les acquis des luttes des divers mouvements, peuples ou individus qui à travers le monde industrialisé ou en voie de développement objectent cette façon d'envisager un nouveau modèle de développement et pensent qu'il n'y a moyen de réaménager la planète que si l'on s'attaque radicalement aux notions de croissance et de besoins et si l'on cesse de tirer un trait d'équivalence entre progrès technique et progrès social. Au demeurant, ces acquis, d'une façon ouverte ou souterraine, continuent à travailler le corps social.

Mais ne serait-ce pas couper court, voire tourner le dos, à un débat autrement plus complexe que de débarquer avec les grandes batteries pointées sur ceux qui se soumettent aux contraintes de cette croissance (contraintes de l'expansion du capital, ajouteront d'autres). En procédant de la sorte, en effet, il n'y a guère moyen de provoquer une discussion politique sur le sujet, c'est-àdire une discussion qui permette de confronter des appréciations différentes sur les rapports de force; on risque donc de répéter ce qu'on a pu constater par exemple à propos de la discussion

8. Actes du colloque national, op. cit., p. 15.

9. Technologie, emploi et croissance (rapport de M.F. Mitterand, président de la République française au sommet des pays industrialisés), château de Versailles, 5 juin 1982. Voir le Monde, 6-7 juin 1982.

10. Actes du colloque national, op. cit., p. 39 . 
sur le nucléaire en maints endroits. Lorsque l'on fait un procès, il faut savoir de quoi on fait le procès. Et ce n'est pas toujours chose aisée.

Comme le souligne le ministre de la Recherche et de la Technologie, «l'objectif de la période actuelle, ce n'est pas le socialisme». Amené à se défendre de ceux qui l'accusaient de transformer le socialisme en une vague référence lointaine, il ajoutait:

Pour ce qui est des socialistes, je ne demande qu'une chose: qu'on les juge sur leurs actes. Le socialisme éclaire notre démarche; c'est l'idéal plein de la démocratie; c'est l'autogestion; c'est la démocratie étendue à tous les aspects de la vie, politique, économique. Cependant, on n'imagine pas que cela puisse se réaliser demain et, à plus forte raison, dans un seul pays isolé du reste du monde. Par conséquent, cela pose bien le problème de l'évolution d'un certain nombre de grands pays et des relations internationales dans la prochaine décennie. Nous devons faire la preuve qu'un mouvement de gauche peut être une réponse à la crise et donc, par un effet d'entraînement, permettre que les choses changent ailleurs, dans d'autres pays $^{11}$.

L'important ici n'est donc pas tant de juger le bien-fondé de la philosophie du développement et du progrès que sous-tendent les objectifs explicites du régime socialiste que de procéder à une mise à plat des contradictions qui les traversent.

\section{L'ÉLECTRONIQUE COMME TECHNOLOGIE DE BASE}

Même si ce thème est loin d'avoir fait l'objet de grands débats publics, c'est sans doute à propos de l'élaboration d'une stratégie industrielle dans le domaine de l'électronique que l'on a pu constater les marges de manœuvre entre lesquelles se meut un politique technologique comme sortie de crise. Nous disposerons ici quelques repères tels qu'ils ressortent des propositions du rapport de la «Mission filière électronique» (rapport Farnoux) remis au ministre de la Recherche et de la Technologie en mars 1982 et présenté à la presse en mai 1982.

1. L'électronique est devenue une technologie de base. Aucun domaine de l'activité économique ne lui échappe. Elle est au cœur des produits stratégiques, depuis les systèmes de défense jusqu'à ceux de communication. La maîtrise de ces produits détermine s'il peut y avoir indépendance technologique, comme élément essentiel de l'indépendance nationale tout court. L'électronique est d'autant plus stratégique que par la nature même de la matière qu'elle traite et véhicule - l'information -, elle est associée à un modèle culturel qui «sera notre modèle ou sera celui imposé par la pénétration de l'électronique étrangère».

2. Sauf à décréter l'autarcie et à isoler la France des changements technologiques du monde, la diffusion massive des produits, services et systèmes électroniques dans l'économie est inévitable. Tout au plus, il est difficile d'en prévoir le rythme. «Le vrai choix est, en réalité, pour notre pays, entre une évolution dans laquelle $i l$ ' $n^{\prime}$ btiendrait qu' une partie des bienfaits du développement de la Filière et tous ses méfaits - parce qu'il se contenterait de consommer, en augmentant inévitablement les importations au détriment de l'emploi national - et une évolution dans laquelle il tirerait le maximum de retombées positives - parce qu'il aurait la volonté de produire ${ }^{12} . »$

3. Tous les secteurs de l'électronique sont interdépendants, depuis le logiciel et les systèmes d'information (dont les banques de données), la bureautique, l'informatique, les automatismes jusqu'aux produits grand public en passant par le militaire. La filière électronique constitue donc un tout et ne peut être traitée que globalement. Cette synergie n'ira que s'accroissant. L'unité de la filière électronique ne fera que se renforcer. «Cette unité - signale le rapport - est d'abord, et sera de plus en plus, technique: la numérisation unifie les informations traitées et homogénéise les composants; entre l'informatique, les télécommunications, la télématique, la bureautique et l'électronique grand public, les frontières s'émoussent, au profit de l'émergence d'un vaste secteur de la communication; au sein des réseaux, la complémentarité se renforce entre les grands équipements - comme les grands ordinateurs ou les satellites - et les petits équipements - comme les microordinateurs ou les centraux téléphoniques privés —; dans l'ensemble de la Filière, le logiciel étend son poids et voit croître son prix relatif. Mais l'unité est aussi, du coup, industrielle, les entreprises étendant vers l'amont ou/et vers l'aval leur champ d'activité ${ }^{13}, »$

11. J.P. Chevènement, Entretien, Témoignage chrétien, Paris, 14-20 juin 1982.

12. Ministère de la Recherche et de la Technologie, Extraits du rapport de synthèse de la mission filière électronique, Paris, Ronéo, mars 1982, Annexe VII, p. 3.

13. Ibid, Annexe V, p. 12 . 
La filière électronique française dans le monde (en milliards de francs)

\begin{tabular}{|c|c|c|c|c|c|c|}
\hline Pays & Production & $\%$ & Marché & $\%$ & $\begin{array}{c}\text { Solde } \\
\text { commercial }\end{array}$ & $\begin{array}{c}\text { Part de la filière } \\
\text { électronique dans le } \\
\text { PIB du pays }\end{array}$ \\
\hline $\begin{array}{l}\text { États-Unis } \\
\text { Japon } \\
\text { Europe de 1'Ouest } \\
\text { dont - RFA } \\
\quad \text { - France } \\
\quad \text { - G.B. } \\
\text { Autres pays' } \\
\text { Monde }\end{array}$ & $\begin{array}{r}668 \\
228 \\
379 \\
113 \\
83 \\
74 \\
175 \\
1450\end{array}$ & $\begin{array}{r}46 \\
16 \\
26 \\
8 \\
6 \\
5 \\
12 \\
100\end{array}$ & $\begin{array}{r}648 \\
164 \\
409 \\
113 \\
82 \\
75 \\
229 \\
1450\end{array}$ & $\begin{array}{r}45 \\
11 \\
28 \\
8 \\
6 \\
5 \\
16 \\
100\end{array}$ & $\begin{array}{l}+20 \\
+64 \\
-30 \\
-1 \\
-1 \\
-54\end{array}$ & $\begin{array}{l}3,5 \% \\
3,7 \% \\
3,3 \% \\
3,0 \% \\
3,8 \%\end{array}$ \\
\hline
\end{tabular}

SOURCE: DIELI, FIEE, Rapport Famoux.

${ }^{1}$ COMECON et Chine exclus.

4. Eu égard à l'unité de la filière, une politique de créneaux n'est pas conseillable pour la France. Il faut une stratégie d'ensemble de redressement de la filière. Cette stratégie s'appuie sur les deux piliers de l'industrie électronique (le matériel professionnel, dont les systèmes d'armes ne sont pas des moindres, les télécommunications couplées avec la télématique) où la France a déjà réussi dans les dernières années à capter une part du marché mondial.

Le redressement des secteurs dits fragiles - les composants par exemple - exige un développement de l'électronique grand public: «L'électronique grand public est le marché naturel des fabriquants de composants. Faute d'une industrie nationale de l'électronique grand public de taille suffisante, jamais les producteurs français de composants ne pourront avoir une compétivité réelle ${ }^{14}$.» La France consacre à la recherche et au développement de produits grand public vingt-cinq fois moins que le Japon.

Recommander une stratégie intégrée ne signifie évidemment pas de «tout faire partout. Une claire conscience des lieux technologiques nodaux, des acquis de la filière française et des effets d'entraînement potentiels permet de préciser les «chantiers» sur lesquels un effort supplémentaire est nécessaire ${ }^{15}, \gg$

Le tableau ci-dessus présente les chiffres d'affaires et le marché de la Filière électronique des États-Unis, du Japon, de l'Europe de l'Ouest et du reste du monde en 1980 (avec un dollar calculé à 5 francs).

5. La stratégie industrielle «filière électronique» proposée pour la France renvoie à une stratégie de décloisonnement de la recherche et de l'industrie qui, comme le faisait remarquer la revue américaine Business Week (31 mai 1982), tire les leçons des expériences de ses concurrents, plus particulièrement le Japon. Avec les nationalisations, les entreprises publiques représentent désormais $49 \%$ de la filière, les sociétés françaises privées y compris les petites et moyennes entreprises représentent $21 \%$ et les groupes étrangers $30 \%$ (dont $13 \%$ pour I.B.M. et $7 \%$ pour Philips). Les laboratoires et centres de recherche nationaux sont entièrement publics. Les recherches, les études et le développement de la filière électronique sont, en conséquence, pour $90 \%$ contrôlés par l'État aujourd'hui.

Sous cette volonté de décloisonnement c'est un nouveau schéma de relations entre l'industrie privée et l'industrie nationalisée, entre industries d'une même branche (pour éviter la concurrence franco-française), entre industrie locale et filiales de sociétés transnationales, entre industrie et université, mais aussi entre constructeurs et usagers qui se dessine. On y décèle à travers le lancement de Projets nationaux flexibles une volonté d'assurer la fluidité des transferts horizontaux de technologie, associant équipes de recherche publiques ou privées, industriels et utilisateurs pour lancer des produits nouveaux. On met au poste de commande la nécessité d'intégrer dans la conception même d'un produit nouveau les notions d'industrialisation et de commercialisation.

14. Ibid, Annexe IV, pp. 7-8.

15. Ibid. 


\section{LA REDISTRIBUTION DE L'ÉCONOMIE MONDIALE}

Cette stratégie industrielle implique de reconsidérer la localisation des entreprises françaises et celle des marchés préférentiels. De par la dimension de leur marché (dix fois la France et la moitié du monde) et leur richesse en technologies, les États-Unis deviennent un lieu prioritaire pour l'internationalisation des firmes françaises.

Parce que le marché européen constitue près d'un tiers du marché mondial, une sortie de crise par la technologie ne peut être atteinte que dans une Europe unie technologiquement. En découle une dynamique d'alliances et de coopération pour la mise au point de nouveaux produits, plus particulièrement des produits grand public.

D'autre part, les possibilités d'automatisation qui répercutent sur le procès de production des produits électroniques «favorise la localisation, voire la relocalisation, dans les pays les plus développés, des productions de la Filière électronique ${ }^{16} \%$.

Il n'y a pas besoin de beaucoup de commentaires pour admettre que c'est sans doute à propos de ces logiques de redistribution de l'économie internationale, inhérentes semble-t-il à un modèle de sortie de crise par la technologie qu'il faudrait s'interroger pour situer la discussion sur le rapport démocratie - technologies de communication. Quelles peuvent-être les conséquences à long terme du double mouvement de relocalisation envisagé - renforcement des liens industriels avec les États-Unis et désengagement probable à l'égard des pays moins développés - sur la formulation d'un rééquilibrage Nord-Sud? Le cadre d'alliances industrielles (qui suit le tracé des alliances militaires atlantiques) ne réduit-il pas à la portion congrue le terrain des négociations économiques avec le tiers monde? Les prises de position occidentales lors des derniers évènements mondiaux tendraient à prouver que l'Europe dans son ensemble croit encore, peut-être erronément, que les contradictions principales passent toujours par la ligne Est/Ouest alors que de plus en plus de pays du tiers monde pensent qu'elles transitent déjà et transiteront chaque jour davantage par la ligne Nord/Sud, et que c'est précisément celle-là qui est en train de réorienter l'axe Est/Ouest. Le chapitre est loin d'être fermé. La rivalité commerciale croissante de la communauté européenne des dix avec les États-Unis est susceptible d'ajouter une autre fissure à un bloc trop bien pensé pour être toujours réel.

Autre question: Quel peut être l'impact des stratégies industrielles des divers pays européens sur cette stratégie industrielle d'indépendance nationale de la France qui ne concorde pas nécessairement avec la vision de chacun de ces pays sur ce qu'est un projet national de réindustrialisation? L'évolution rapide des modèles de l'économie néo-libérale dans des pays comme la GrandeBretagne qui propulse ce pays au rang d'avant-garde européenne d'un modèle de développement calqué sur la logique transnationale n'est-elle pas susceptible d'interférer dans le déroulement d'une stratégie nationale qui, à travers de nombreuses contradictions, tente d'échapper à l'emprise japonaise ou nord-américaine? La rapidité avec laquelle s'installent les nouvelles technologies de communication (le Royaume-Uni devrait disposer, par exemple en 1983 du premier réseau privé de télécommunication par fibres optiques, en Europe, en compétition directe avec les PTT nationaux), mériteraient que l'on s'étende sur ces nouvelles données qui constituent le cadre concret dans lequel prend issue et forme le progrès technique en Europe. Un indice, parmi d'autres, de ce rôle de tête de pont joué par la Grande-Bretagne est celui des flux internationaux de données. $66 \%$ de tous les flux de données de l'Europe occidentale vers les États-Unis ont leur point de départ au Royaume-Uni. Les flux de données transatlantiques, en fait, représentent deux tiers des flux transfrontières de données de ce pays, contre une moyenne de $10 \%$ pour tous les autres pays de l'Europe occidentale ${ }^{17}$.

On sait par ailleurs, que les grandes firmes électroniques ont dans les années 77-78 choisi comme cibles prioritaires pour le lancement du magnétoscope grand public en Europe, d'abord la Grande-Bretagne et ensuite l'Allemagne fédérale. Des raisons multiples, insuffisamment analysées à notre sens, les ont poussé à agir de la sorte. Raisons qui tiennent tout autant aux habitudes et aux modalités de consommation audiovisuelle (par exemple l'importance des réseaux de location d'appareils en Grande-Bretagne comparés à ceux de la France) qu'à la structure de l'industrie de

16. Ibid, Annexe V, p. 12. Rappelons quelques unes des orientations du programme concerté de croissance par la technologie proposé par F. Mitterand au Sommet de Versailles: «Des actions prioritaires de coopération technologique entre firmes privées et publiques et entre nations...; création progressive d'un marché mondial de technologie (normes, brevets); ... initiatives conjointes pour assurer aux Pays du Sud la maîtrise des nouvelles technologies». Voir op. cit.

17. Nations unies, Transnational Corporations and Transborder Data Flows: A Technical Paper, New York, U.N. Centre on Transnational Corporations, 1982. 
la production de téléviseurs (dans les deux pays choisis comme cibles préférentielles, une industrie nationale extrêmement dispersée qui a d'ailleurs été décimée à l'occasion de l'arrivée des magnétoscopes); raisons également qui tiennent à la nature du système télévisuel de chacun de ces pays. Bref, pour pouvoir circonscrire les facteurs et éléments qui sont intervenus dans le cas du magnétoscope, c'est à une histoire comparative, quasi inexistante, des systèmes de communication en Europe qu'il faudrait avoir recours; histoire qui devrait nous expliquer pourquoi la Grande-Bretagne, depuis l'essor de la radio a devancé tous les autres pays européens dans l'absorption des technologies audiovisuelles. Ces études comparatives sont un chemin obligé pour tous ceux qui tentent de répondre à la question essentielle: pourquoi en 1962 la France était-elle un des pays européens où le parc de téléviseurs était le plus restreint: $27 \%$ des foyers possédaient un poste contre $29 \%$ en Italie, $37 \%$ en Belgique, $41 \%$ en Allemagne fédérale, $50 \%$ aux Pays-Bas et $82 \%$ en GrandeBretagne. Elles devraient également permettre de répondre à cette autre question: pourquoi constatet-on aujourd'hui un décalage si important entre les divers pays européens par rapport au parc de télévisions en couleur? Si on ne veut pas se contenter d'une réponse mécaniste et unilatérale qui consisterait à renvoyer à une analyse de la progression des revenus, c'est à une recherche complexe qu'il faudrait s'attacher.

\section{DES PÔLES DE PRODUCTION CULTURELLE?}

D'une façon ou d'une autre, quelques uns des axes problématiques qui sillonnent l'option proposée pour l'ensemble de la filière électronique, ce que l'on convient d'appeler les «industries du contenant», se retrouvent avec leur spécificité propre lorsqu'il s'agit de formuler une stratégie pour les «industries du contenu». Il ne peut en être autrement puisque la dynamique de ces dernières s'inscrit aussi dans un même mouvement d'unification. Mouvement d'unification dont il n'est plus besoin de faire la preuve autant dans le champ de l'information - marchandise, où la logique de la chaîne solidaire des services électroniques pousse à l'intégration des différents intervenants (serveurs-producteurs-transporteurs) que dans le champ du divertissement qui tend à se confondre avec le premier (conglomérats multimedias, etc.). Programmes, logiciels, architectures de réseaux, l'enjeu est tout à la fois économique, industriel, social, et culturel.

Mais s'il est relativement aisé d'ébaucher les lignes de force d'une stratégie de développement des technologies électroniques - même si celle-ci n'est encore qu'en phase de gestation -, il est beaucoup moins facile de dégager les linéaments d'une stratégie équivalente en matière de production culturelle.

Que le problème soit posé de la nécessité d'articuler divers partenaires pour susciter une réponse nationale face à l'hégémonie des productions transnationales (séries de télévision, jouets, vidéo-cassettes, etc.), cela est indubitable. Mais les véritables divergences commencent lorsqu'il faut déterminer de quels partenaires il s'agit. Écoutons le directeur chargé des activités nouvelles de Hachette, la cinquième entreprise mondiale d'éditions:

Du fait de sa position Hachette s'est trouvée, parmi les premiers, impliquée dans une mutation dont les enjeux concernent l'ensemble des intervenants du secteur culturel. Les handicaps de la production et de la distribution audiovisuelles françaises, vis-à-vis de celles des ÉtatsUnis, sont tels que le défi se pose moins en termes de concurrence entre les éditeurs ou les producteurs français, qu'en termes de solidarité et de complémentarité. C'est là une des dimensions de l'action à laquelle nous nous sommes associés. Nous souhaitons que la concertation engagée avec quelques-unes des principales sociétés françaises de production audiovisuelle, pour établir les bases d'une politique d'édition dans ce domaine (notamment au plan juridique), puisse se développer et s'étendre à d'autres sociétés, et à d'autres éditeurs ${ }^{18}$.

Mais les obstacles à la réalisation effective d'une synergie entre divers éditeurs ou producteurs sont autrement plus complexes que lorsqu'il s'agit d'entraver la concurrence franco-française en matière d'électronique. L'idée du rapprochement entre un secteur public massivement présent dans l'audiovisuel et les grands groupes privés n'est pas évidente. Non seulement ces grands groupes privés ont une expérience très limitée de la production télévisée à cause de la domination du secteur public mais surtout leurs relations avec l'État sont conflictuelles. Et ceci pour des raisons bien précises sur lesquelles il faudra revenir mais qui sont suggérées par le responsable du développement des nouvelles technologies de Havas, grand groupe multimedia partiellement contrôlé par l'État.

18. Dans Livres-hebdo, Paris, 22 septembre 1981. 
Tout ce qui n'est pas du secteur public souffre en France d'un manque réel de légitimité symbolique dès qu'il s'agit d'audiovisuel. Il n'est donc pas surprenant que les négociations entre l'État et les sociétés privées, ou même d'économie mixte, soient si conflictuelles dès qu'il est question de gérer des activités audiovisuelles. Accusés de n'avoir pour but qu'un développement commercial reposant sur une logique de marché dont on connaît les dangers dans ce domaine, les groupes de communication français ne peuvent affirmer aucune ambition réelle. La place reste donc libre pour d'autres groupes transnationaux qui lancent déjà des opérations dans l'édition (Bertelsmann, Pergamon, Time-Life) avant d'attaquer le domaine audiovisuel en utilisant les processus de dérèglementation que l'on voit se développer dans plusieurs pays européens ${ }^{19}$.

Or selon le même responsable de Havas* qui s'exprime évidemment à titre personnel, la recherche d'une nouvelle légitimité pour les activités de diffusion audiovisuelles hors du service public «ne peut passer que par une action de soutien volontaire et raisonnée des potentiels de création qui existent en France dans ce domaine». Ce type de diagnostic amène à proposer la constitution d'un nouveau soubassement socioindustriel, il recommande de s'adosser à quelques pôles complémentaires de création et de production (Hachette, Havas, Sofirad, les radios périphériques, principalement Europe 1, et l'appareil télévisuel) pour une croissance susceptible de rivaliser auprès des audiences internationale ${ }^{20}$ et de tisser autour de ces pôles de nouveaux rapports entre partenaires multiples.

Pour des raisons connues de tous, les petites structures audiovisuelles, novatrices et ambitieuses, rencontrent de multiples difficultés pour se développer. Partout des créateurs, des journalistes qui portent en eux ce que sera la presse de demain, des techniciens capables d'innovations remarquables (logiciels, dispositifs haute fréquence, haute fidélité, vidéo) cherchent les moyens de réaliser ce dont ils sont porteurs. Donner aujourd'hui leur chance à ce genre d'initiative, c'est évidemment le rôle de l'État, mais aussi celui des grandes entreprises du domaine. C'est une initiative susceptible d'avoir des retombées commerciales et qui est aussi une manière privilégiée de conquérir une légitimité auprès d'une opinion publique toujours favorablement impressionnée par les actions dans ce domaine ${ }^{21}$.

Les propos des responsables des chaînes de télévisions vont dans le même sens: grâce à la nouvelle loi de l'audiovisuel ils espèrent eux aussi trouver d'autres partenaires:

Je souhaite - déclarait en mai 1982 le Directeur de programme de la première chaîne (TF 1) - aussi pouvoir créer, avec différents partenaires une filiale de diversification, afin de démultipliier nos possibilités de programmateurs. La chaîne devrait être présente sur tous les marchés de la création audiovisuelle (la télédistribution, le vidéo-cassette...) en lien direct avec les industriels ${ }^{22}$.

L'évolution de ce schéma d'alliances (grand/petit, national/local, appareils centraux/périphérie, privé/public, commercial/associatif) sera déterminante, car elle conditionnera le type de rapports

19. A. Lefébure, «Mass media, groupes de communication et société», contribution personnelle à la mission «Technologie, diffusion de la culture et communication», juin 1982.

* En mars 1983, Havas a reçu la mission de préparer le projet de la quatrième chaîne de télévision. D'autre part, la consolidation de groupes multimédias s'est accentuée fortement depuis mai 1981.

20. Quelques chiffres pour situer l'internationalisation des industries culturelles françaises. En 1980, les ventes de films français à l'étranger ne représentaient que le dixième des ventes de livres et la moitié des ventes de disques français. Quant à la vente de programmes de télévision, elle ne représentait, elle-même que le cinquième de la vente de films. Les ventes de la télévision à l'étranger représentaient environ un tiers des achats à l'étranger. Encore faut-il savoir que les exportations de l'industrie de l'édition représentaient à la même date moins de $20 \%$ de son chiffre d'affaires. Et en outre étaient surtout centrés sur les pays francophones qui absorbaient plus de $80 \%$ de ce courant d'exportation. (voir J. Rigaud, les Reiations culturelles extérieures, Paris, La Documentation française, 1980). En 1980, l'appareil télévisuel français (TF1, A2, FR3 INA -vente commerciale-, SFP -cession de droits-) a vendu à l'étranger 5596 d'heures-programme contre 1567 en 1978. En 1980, également, 6000 heures-programme TV ont été envoyées, à titre d'assistance culturelle, à 29 pays dont 15 en Afrique noire ( 3500 heures) et le reste au Maghreb et à Haïti, et dans quelques pays arabophones ou anglophones. Quant aux coproductions elles se sont élevees à 94 heures pour TFl et 36 heures pour A2 en 1980. (Pour FR3, les coproductions sont estimées pour la période 1979/80 à 60 heures). Par ailleurs, les coproducteurs restaient les partenaires traditionnels (francophones et RFA) sauf pour FR3 (Japon, Mexique) (chiffres provenant du rapport du groupe de travail «Action internationale» de la commission Moinot, août 1981).

21. A. Lefébure, doc. cité.

22. «Entretien avec André Harris; le déficit de l'imagination», le Monde, 27 mai 1982. 
de force entre des partenaires, de poids inégaux, qui n'ont pas nécessairement la même conception de l'indépendance nationale ni non plus la même idée de démocratie en ce domaine...!

\section{L’ÉTAT DANS TOUS SES ÉTATS...}

Pauvre État! On lui demande tant. Et des tâches pour le moins difficilement conciliables: on lui suggère non seulement de s'ériger en arbitre, de venir à la rescousse du sommet, c'est-àdire, des grandes entreprises en mal d'esprit d'entreprise mais également d'appuyer toute la base de la pyramide, tous ceux qui font dans le petit, tous ceux qui rendent crédibles les nouveaux réseaux de solidarité. Il serait donc peut-être temps de se demander de quel État il s'agit. Car c'est précisément là que se situe la question essentielle pour cerner le rapport technologie-communicationdémocratie.

Dans la plupart des esprits, le 10 mai a signifié pour la France, sous de nombreux aspects, la possibilité de se soustraire à des logiques en oeuvre dans la plupart des grands pays capitalistes avancés. Pour réussir sa mutation au sein de la restructuration de l'économie mondiale, la France aurait depuis la victoire socialiste mis entre parenthèses ces grands mouvements de fond qui continue à effecter ses voisins ou ses partenaires. Et plus particulièrement elle pourrait ne pas se poser ces questions lancinantes auxquelles doivent s'affronter les forces néo-libérales de l'Angleterre et des Etats-Unis par exemple, obligées pour les besoins de la cause de se métamorphoser en «penseurs de l'État». La France n'aurait que faire de ces logiques de privatisation qui entament le Welfare state (l'État providence) puisque le projet socialiste aurait décidé de lui donner un second souffle.

Or rien n'est plus faux que de croire que ces logiques ont disparu par le seul fait d'une victoire aux urnes. Elles continuent à travailler la société française, avec des modalités propres qui remettent au goût du jour des problématiques de fond.

1. De nombreux indices pris dans le domaine de la communication attestent l'accélération du procès de redistribution du mode de gestion du rapport public/privé dans les dernières années soixante dix.

Pour faire face à une perte de légitimité, l'État, et sa logique administrative, ont cherché des relais auprès des procédures en vigueur dans le privé. Tandis que le privé repensait le «social» à partir des finalités de l'État. Pour enrayer la crise de l'État, qui est aussi une crise d'image, on a appelé à la rescousse le management qui offrait à cet État une façon de se rationaliser, c'est-àdire d'éviter les gaspillages, ainsi que le marketing qui engageait une autre relation avec le public. On assista alors à la multiplication de la «communication publique». C'est ainsi que la dépense totale pour l'achat d'espaces publicitaires dans les médias par l'État est passée de 39,7 millions de francs en 1977 à 62,3 millions en 1979 dont la moitié pour le seul ministère du travail et de la participation. Pour comparer ces chiffres il faut les corriger en tenant compte des rabattements de tarifs consentis aux campagnes qualifiées d'intérêt général (pour la télévision, les administrations ne paient que le quart du barême commercial). Ce qui correspondrait à un budget de 128 millions de francs, chiffre sensiblement voisin des deux ou trois plus gros annonceurs privés: L'Oréal, Colgate-Palmolive ou Unilever ${ }^{23}$.

Un deuxième indice de poids est celui de l'offensive télématique amorcée à partir de 1976. Cette offensive avec comme maître d'œuvre la Direction générale des télécommunications (DGT) du ministère des PTT a signifié sans aucun doute une entrée en force des schémas managériaux dans la gestion de l'État. Cette logique commerciale qui a accompagné la promotion des nouvelles technologies a fait sentir ses effets aussi bien dans l'approche du marché intérieur que dans celui, fondamental, des marchés internationaux (plus particulièrement dans les grands pays latino-américains). Comme on a très peu analysé la transformation des pratiques d'État de ce point de vue, il est difficile de situer avec rigueur les disfonctionnements et les asynchronismes que ne manque pas de provoquer dans une partie de l'appareil d'État - ici le ministère des PTT — la modernisation de certaines de ses divisions. Car il serait faux de croire que ce que l'on devrait appeler à juste titre le procès de marchandisation des actions administratives pénètre de façon uniforme l'appareil d'État. Des résistances de tout type (depuis celles qui prennent appui sur la défense du service

23. Sur la crise de l'image de l'État voir les diverses contributions au colloque «où va la gestion publique» (2830 mai 1980) organisé par l'université de Paris-Dauphine et le centre d'enseignement supérieur des affaires (CESA). Voir plus particulièrement les contributions de J. Lendrevie et R. Laufer. Voir également J.L. Albert et. al., Production de la ville et aménagement du discours, Grenoble III, GRESEC, janvier 1980, ronéo. 
public jusqu'à celles qui se prévalent des corporatismes institutionnels ou professionnels en passant par celles qui émanent de la terra incognita des usagers) s'interposent produisant bien souvent conflits et décalages.

Une autre illustration du réaménagement du rapport public/privé est fournie par ce qui devait se révéler être la première grande opération française de rapprochement entre un fabricant d'équipement lourd, la firme électronique Matra, et le fabricant de programmes (Hachette). Il est en effet difficile d'interpréter cette fusion qui se réalise fin 1980 sans tenir compte des pressions subtiles d'une action volontariste de la part du gouvernement du président Giscard d'Estaing soucieux de redéfinir l'alliance entre l'État et les grands groupes multimedia au seuil de l'arrivée des nouvelles technologies.

Enfin, de façon plus globale, la dispute idéologique (si tant est qu'il y eut débat), autour du rôle des intellectuels (et de leur rapport avec les médias) a indiqué elle aussi à sa façon une rupture épistémologique. Sous l'éphémère épiphénomène des «nouveaux philosophes» inséparable de leur écho médiatique, c'était un nouveau mode de diffusion (et donc de production) du savoir qui se profilait à l'horizon au moment même où la loi de la valeur accentuait sa percée dans le champ intellectuel. Au-delà de l'anecdote, ce fut, parmi d'autres, un des premiers indices de la nécessaire redéfinition du rapport entre la petite bourgeoisie, les gestionnaires du savoir, (dont on n'a pas assez scruté la diversité parce qu'on a trop vite consacré un concept étroit et peu adapté aux nouvelles conditions historiques d'intellectuel), l'appareil d'État et la logique du marché24.

2. Mais la légitimité de l'État a également dans les dernières années 70 été mise à rude épreuve par d'autres forces. Ont été secoués non seulement le rapport entre l'État et la société civile mais également les différents modes d'action des divers composants de cette société civile.

D'une part, les formes de légitimité de l'État à partir du centre se sont de plus en plus effritées. La revendication de décentralisation est devenue un lieu d'affrontements sociaux et donc d'ambiguittés. En se «décentralisant», certains secteurs ont pensé y trouver de nouvelles formes de légitimation du centre à partir de la périphérie; d'autres ont vu dans ce retour au local, au spécifique, un moyen d'approfondir la démocratie dans un sens de partage réel du pouvoir. C'est autour du «local» que se sont noués de nombreuses luttes mais également de nombreux fantasmes sur les vertus démocratiques per se de toute forme de décentralisation ${ }^{25}$.

Dans ce contexte est apparu, entre autres, le mouvement des radios libres qui a son équivalent dans des mouvements qui recouvrent d'autres formes d'expression, moins publicisées parce que moins nouvellement techniques.

À travers cette interrogation sur le fonctionnement de la société civile, c'est aussi une conception monolithique et manipulatoire de l'État qui s'est ébranlée. L'État n'est plus exclusivement perçu comme ce lieu de reproduction tautologique du pouvoir mais comme lieu de production du pouvoir. Un lieu marqué par des rapports de force entre groupes, classes et projets sociaux où se jouent certes l'affirmation d'une hégémonie mais aussi des stratégies de contournement et de détournement. À travers cette interrogation, c'est aussi une façon de concevoir l'action des partis et le rapport avec leurs militants dont on entendait faire le procès

\section{LA RELATION PUBLIC/PRIVÉ}

Qu'en est-il aujourd'hui de ces mouvements de fond?

La tendance à confier au secteur publicitaire les grandes campagnes d'intérêt général s'est poursuivie comme en témoigne la première campagne pour développer la lecture. Marquée par la diffusion de spots intitulés: «la lecture: les chemins de la liberté», programmés deux mois durant sur less écrans de télévision, la campagne a été relayée par une opération d'affichage, chez les libraires. Le chiffre consacré à ces campagnes a même fortement augmenté: en 1982, on estime qu'il devrait s'élever à 150 ou 160 millions de francs (toutes taxes comprises) comparés aux 62 millions de 1979. Avec raison, on peut se demander - comme le fait Bernard Miège - si «avec la cornmunication publique on n'assiste pas au renforcement d'un processus de gestion de l'opinion publique, d'autant plus dangereux qu'il prend la place de procédures démocratiques de débat ${ }^{26} »$.

24. Voir Régis Debray, le Pouvoir intellectuel en France, Paris, Ramsay, 1979; F. Aubral et X. Delcourt, Contre la nouvelle philosophie, Paris, Gallimard. 1977.

25. Voir l'Objet local, colloque dirigé par Lucien Sfez, Paris, collection 10/18, 1977.

26. B. Miège, «Le pouvoir et les systèmes d'information: s'interroger sur les enjeux fondamentaux», contribution au troisième congrès français des sciences de l'information et de la communication, mai 1982, ronéo. 
Tant il est vrai qu'à force de demander trop au marketing, on en oublie de demander à la société civile les dispositifs d'accompagnement qui permettraient d'ancrer dans la réalité de chaque citoyen une prise de conscience sur les enjeux de la lecture, de la contraception, de la solidarité, etc. Là encore, la rentabilité d'une technique (ici pour le lancement d'un produit dit social) semble admise $\bar{a}$ priori et l'on ne s'interroge guère sur les formes verticales de relations sociales qu'elle perpétue. C'est un point de plus qui vient s'ajouter à d'autres dans les dérives technicistes.

Mais le débat sur l'État et sur le rapport privé/public dans le champ de la communication est autrement plus complexe aujourd'hui qu'il ne l'était il y a un peu plus d'un an. Les propos des responsables d'Hachette et surtout d'Havas le laissaient déjà soupçonner. Et plus concrètement pour qui est confronté quotidiennement à la recherche d'alternatives démocratiques, la façon de vivre le rapport public/privé est devenu plus conflictuelle. Car, on ne le dira jamais assez; il existe en France une véritable «culture du public», vécue comme culture légitime, culture d'excellence, par de nombreux secteurs, plus particulièrement ceux dont la montée sociale s'est faite en s'appuyant sur l'appareil étatique ou paraétatique. Cette culture qui a son envers ostracisé, - un véritable délire contre le privé - n'est pas un des moindres obstacles à repenser l'alliance de la science, de la recherche et de l'industrie, tel que l'a proposé le colloque national. D'autant plus que cette culture est construite sur un paradoxe. À travers cette «culture du public», le rapport à l'État se vit sur un mode sado-masochiste. C'est ce même État dont on exige qu'il exerce ses fonctions de protecteur et d'arbitre qu'on n'hésitera pas à accuser de dirigisme avec d'autant plus d'agressivité que l'on en est plus redevable. Dans le domaine de la production symbolique les passerelles pour une discussion critique sur des enjeux d'un rapprochement public/privé sont encore plus rares car cette «culture du public» y est curieusement croisée avec une conception hyper-individualisée et esthétisante de la création - ce qui se traduit chez certains par une méfiance viscérale à l'égard de tout ce qui peut donner origine à une matrice industriellement reproductible. Toutes réticences d'ailleurs dont il est difficile de départager les incidences positives et négatives aussi bien sur le processus actuel d'internationalisation des industries culturelles, que sur la mise en place des mécanismes de résistance à la normalisation des modes de vivre, de penser et de créer.

Les demandes latentes à l'égard d'une politique officielle d'accompagnement formulées par certains grands groupes multimedia attestent sans aucun doute d'une évolution dans le rapport entreprise privée/État. Mais on ne peut la saisir que si on la resitue dans un mouvement plus vaste de changement des rapports entre l'entreprise privée et l'ensemble de la société, et plus particulièrement dans le domaine qui nous occupe, avec les sources vives de la création et de l'innovation sociale. Comment interpréter autrement cette «lettre ouverte aux innovateurs», publiée à pleine page dans le Monde du 17 février 1982, adressée à «tous les innovateurs, créateurs de logiciels ou de matériel dans tous les secteurs: micros individuels, jeux programmables, gestion, bureautique, télématique ou calcul scientifique» par Matra-Hachette:

Le développement de la microinformatique grand public est irrévocable. L'enjeu économique, culturel, social est considérable. Malgré la pression concurrentielle étrangère, la France a les moyens d'occuper une place prépondérante dans ce secteur. Cette position ne sera acquise que si le dévelopement de la microinformatique Grand Public s'intègre dans une stratégie définie. Et s'appuie sur des structures industrielles et commerciales éprouvées. La stratégie est définie. Les structures existent. Par sa maîtrise des technologies de pointe - du composant aux télécommunications - Matra rassemble le potentiel technique et industriel nécessaire au développement d'une véritable industrie française de la Micro-informatique.

Par son expérience dans le domaine de la communication écrite et de sa structure de diffusion, Hachette apporte les conditions complémentaires indispensables à la réussite de cette entreprise. C'est pourquoi Matra et Hachette ont décidé, ensemble, la création d'un groupe de recherche, de développement et de distribution dans le domaine de la microinformatique de grande diffusion.

Ce groupe constitue une structure «d'accueil». Pour tous ceux - personnes ou entreprises - qui ont l'esprit imaginatif, créatif. Ceux qui peuvent et qui veulent nous aider à placer la microinformatique française aux premiers rangs de la compétition internationale.

Pour qui veut bien dépasser la problématique promotionnelle de la simple captation de créateurs individuels, il y a là matière à réflexion. Comme nous l'écrivions récemment en une autre occasion, tentant de généraliser à partir d'observations prélevées dans d'autres réalités nationales:

Si la question du rapport entre la dite industrie culturelle et le reste de la société (institutions, associations, groupes, individus) comme source de créativité pour alimenter la production 
a toujours occupé une place importante dans le fonctionnement des divers secteurs de cette industrie, il va sans dire que la pénétration croissante du capital dans les sphères les plus diverses de la vie quotidienne des collectivités et des individus par le truchement des systèmes informatisés risque de faire de ce rapport industrie-société civile un enjeu global prioritaire. La prospection des usages sociaux pour les nouvelles technologies d'information pose avec acuité dans des termes politiques et économiques qualitativement différents de ceux connus jusqu'alors le lien entre «l'industrie de l'information» et les lieux de l'innovation sociale. De nouvelles constellations de rapports entre partenaires industriels et non industriels sont en train de se dessiner reflétant dans chaque réalité des rapports de force spécifiques ${ }^{27}$.

La façon dont les firmes informatiques transnationales essayent en divers points du monde de contourner les résistances des appareils d'enseignement nationaux, est sur ce point déjà très révélatrice, et de ce point de vue, on ne peut que s'inquiéter de l'extrême fluidité des sociétés transnationales et de leurs stratégies «portes ouvertes aux expérimentations» qui contrastent souvent avec l'extrême rigidité des appareils institutionnels.

Tout ceci conduit à s'interroger sur la façon spécifique dont chaque société nationale organise, met à profit les innovations culturelles (ou y résiste), et gère sous cet angle particulier sa transition à l'ère électronique. Il y a peu, le directeur des programmes de la première chaîne (TF 1) lamentait le manque de réflexion sur les questions posées par l'évolution de la technique:

Les ingénieurs et les techniciens ont pris une avance inouie sur les gens de programme, et

l'on se gargarise du câble et du satellite sans comprendre qu'à de nouveaux médias devront correspondre de nouveaux programmes. Peu de pays ont mené dans ce domaine une politique prospective, et nous souffrons aujourd'hui d'un véritable déficit de l'imagination ${ }^{28}$.

Déficit d'imagination qui ne se confond pas nécessairement avec un manque de sources de créativité. Un pays peut très bien regorger d'idées mais ne pas disposer de structures qui permettent leur mise en œuvre 29 . Cela renvoie à l'histoire, particulière à chaque nation, des formes qu'a assumé l'expansion du capital dans la sphère culturelle ainsi qu'à celle des modalités de l'articulation entre les créateurs et l'ensemble des appareils de production culturelle (privés ou publics).

\section{LA DEMANDE SOCIALE ET LA RECHERCHE}

Une préoccupation a traversé le colloque de la recherche et s'est cristallisée dans une notion aux contours imprécis: la demande sociale. On a parlé du «retard social de la recherche», on a osé parler du fossé qui s'est creusé entre les recherches et les demandes des secteurs populaires. Nous n'hésiterons pas à citer un long extrait du rapport de la première commission:

Encore faut-il que les objets auxquels les scientifiques consacreront leurs efforts soient choisis de façon pertinente. L'État dira ses priorités, à commencer par la plus évidente d'entre elles, qui est d'aider notre pays à sortir de la crise. Les industries, mieux irriguées de savoirs et de savoir-faire nouveaux, révèleront de nouveaux besoins. Les scientifiques eux-mêmes, trouveront selon la logique de leurs activités, de nouvelles questions, de nouveaux champs

27. A. Mattelart, «Introduction», Communication and Class Struggle, New York, International General, t. II, sous presse.

28. «Entretien avec André Harris», art. cit.

29. Un exemple: on peut penser ce que l'on veut de programmes comme Ulysse 31 comme modèle de télévision pour enfants mais l'histoire de sa production est, d'un point de vue industriel, fort illustrante. Conçue par un français, la série n'a pu être réalisée en France et après bien des avatars, comme la plupart des séries animées, a été produite au Japon grâce à un accord de coproduction. Or, dans ce cas comme dans beaucoup d'autres, comme l'a observé le groupe de réflexion sur la politique de l'image mis sur pieds par le premier ministre, les éléments sont présents sous forme éparse dans des laboraloires universitaires, des industries électroniques, mais le transfert horizontal de technologies et de talents ne s'opère pas. Ce qui fait écrire au responsable du département des émissions pour la jeunesse de TF1 convaincu de ce que l'État ne doit pas créer lui-même une industrie du dessin animé, mais seulement les conditions pour que celle-ci puisse s'épanouir et se développer: «un millier de professionnels ont le talent et les capacités pour faire entrer véritablement le dessin animé frança is dans le monde d'une télévision moderne, à condition de ne pas rester des artisans isolés, à condition d'être soutenus et enciadrés par des managers rompus aux règles de cette industrie nouvelle, à condition surtout que soit ranimée par des mesurts concrètes la flamme de leur foi». (J. Mousseau, «Plaidoyer pour une industrie française du dessin animé», Communication et lanł̧ages, deuxième trimestre $\left.1982, \mathrm{n}^{\circ} 52\right)$.

Plus significatif encore d'un gaspillage ou d'une non utilisation d'un potentiel est sans doute le cas de l'annuaire officiel des abonnés du téléphone sur lequel les autorités semblent avoir peu réfléchi en France tant du point de vue de la lisibilité que de celui de la sémiologie alors que les PTT italiennes et belges utilisent la créativité d'une des rares équipes de graphistes et sociologues existantes dans le monde - l'équipe française (de Ladislas Mandel) - en leur confiant la réalisation de leur annuaire. Voilà un cas où la sémiologie pourrait aller à la rencontre d'une «demande sociale» insoupçonnée! 
d'expérimentation, de nouveaux paradigmes théoriques. Mais ces trois démarches - qu'il faut d'ailleurs combiner mieux — seront loin d'épuiser toute la demande sociale à expliciter et à satisfaire.

Le fait est que les syndicats, les associations et les mouvements sociaux encore faiblement appareillés (qui se nomment: écologie, féminisme, organisations de consommateurs, etc.) en disposent présentement ni de l'autorité ni des ressources qui leur permettraient d'adresser de nouvelles demandes aux institutions scientifiques ou de développer leurs propres recherches - et pourtant, il faut faire droit à leurs besoins. Le fait est que la décentralisation, désormais engagée, va susciter, avec la maturation de nouveaux centres de décision, l'émergence de nouvelles demandes qu'il conviendra de satisfaire, voire de prévenir. Le fait est que de vastes pans de la vie sociale font encore l'objet d'insuffisantes investigations scientifiques, qu'il s'agisse de la vie quotidienne, de la condition inégalitaire faite aux femmes, de la jeunesse, mal insérée dans notre société, de la pédagogie qui tarde à trouver un nouvel équilibre, des conditions de travail, sans cesse modifiées par les nouvelles technologies, des villes qui ont proliféré, des arts où fermentent de nouvelles curiosités scientifiques, etc., et il faudra donner corps aux recherches portant sur de tels objets, fût-ce au prix de réorganisations institutionnelles majeures. Le fait est que la distance est grande - et, pour tout dire, excessive - entre le savoir-faire qui s'exerce dans l'ordre politique et le savoir que les sciences sociales accumulent, sans s'intéresser suffisamment aux savoir-faire politiques qu'elles pourraient éclairer. Plus généralement, le fait est que la recherche ne laboure qu'une partie du champ qu'elle devrait travailler.

Si bien qu'il importe de réduire cet écart par tous les moyens: en aidant de nouvelles démarches à s'exprimer, en favorisant l'exploration des besoins latents par les institutions scientifiques elles-mêmes, en multipliant les contacts et les expériences, en établissant des relais sociaux entre les demandes éparses dans notre peuple et les agences spécialisées dans la recherche. Bref, en essayant de rattraper le retard social de la recherche.

Des erreurs, des échecs et des tâtonnements sanctionneront sans doute cette ambitieuse poussée de nouvelles recherches répondant à de nouvelles demandes. Mais nul ne peut douter que deux succès sont néanmoins à notre portée: l'un sera un succès par la science, éclairant une partie au moins du domaine à conquérir; l'autre sera un succès pour la science, dans la mesure où elle donnera à la société tout entière de nouvelles preuves de son utilité sociale ${ }^{30}$.

Les sciences de la communication telles qu'elles se sont construites en France n'échappent pas au tropisme dont souffrent les autres sciences sociales. Statut hypertrophié pour certaines problématiques de recherches, statut minorisé pour d'autres, dans un contexte où les études sur la communication occupent un strapontin dans l'ensemble des sciences sociales, économiques et politiques. La liste des carences en fonction de cette idée de «demande sociale» serait longue à dresser. Quelques exemples de lacunes (dans ces cas, tout ou presque reste à explorer):

Absence de relations entre les recherches en sémiologie et la demande latente, bien réelle, d'analyses du discours de la part des journalistes en quête d'une redéfinition de leur pratique. Lacunes qui contrastent avec la fluidité des échanges entre les recherches sémiologiques et l'industrie publicitaire.

- Absence de connaissances précises sur les modes de ré-appropriation du discours médiatique par les différentes catégories sociales qui composent le «grand public».

- Absence d'analyse des stratégies de détournement et de contournement des dispositifs du pouvoir pratiquées par les multiples acteurs sociaux.

- Absence d'études qui se soucient d'articuler, dans un modèle d'analyse dialectique, les expériences dites d'intervention sociale (cinéma, vidéo, radios) et le fonctionnement des appareils centraux, les expériences académiques en matière audiovisuelle et leurs retombées pratiques. Que l'on pense par exemple à l'importance du film scientifique (à travers les travaux de Painlevé) dans le développement de l'avant-garde cinématographique française.

- Absence d'analyse dialectique de l'échange ou du manque d'échange entre les recherches universitaires (par exemple en matière de cinéma) et le champ de la critique (dans les journaux, revues, magazines, etc.) D'une façon ou d'une autre, dans beaucoup de domaines, malgré l'apparente déconnection, les références et les acquis de la recherche académique fixent les seuils de tolérance d'un discours. La critique profane - ou du moins un secteur d'entre elle - ne se permet plus dans le traitement des films ce qu'elle se permet encore dans le domaine de l'analyse de la musique dite populaire (rock, variétés, etc.). L'absence notoire de recherches en ce domaine (il n'existe en France pratiquement qu'un ou deux travaux de poids sur l'industrie culturelle musicale et encore

30. «Commission $n^{\circ} 1$. L'apport culturel de la recherche scientifique et technologique» (rapporteur: Robert Fossaert). Actes du colloque, op. cit. pp 92-93. 
ne sont-ils pas inspirés par une vision antropologique du phénomène de la musique populaire), rend crédible les discours les plus mystifiants et idéalistes dans les grands reportages médiatiques. Dans la même perspective, on ne peut échapper à la question de savoir comment dans un pays où le commercial/privé ne jouit pas d'une légitimité symbolique élevée, les références académiques sont pour un milieu de recherche privée qui vit mal son rapport au profit de puissants outils de légitimation et de dédouanement à l'égard de la logique du marché.

- Absence, pour ainsi dire, d'études sur le rôle des créateurs, leur rapport avec les industries culturelles, leur rapport avec la société civile.

- Et surtout, absence d'un bilan critique des multiples expériences de communication alternative qui depuis près de quinze ans ont travaillé le corps social.

- Ces lacunes, d'aucuns diront cette surdité de la recherche, sont difficilement explicables si l'on ne tient pas compte des obstacles institutionnels qui ont cloisonné les recherches mais aussi et surtout des grands courants de réalité et de pensée qui ont agité et modulé un type de rapport avec l'ensemble de la société.

\section{L’«EXPÉRIMENTATION SOCIALE» COMME ENJEU}

À l'intersection des exigences d'une dynamique industrielle de développement des nouvelles technologies et de la nécessité d'associer à la mise en ouvre de celles-ci les partenaires sociaux qui en étaient écartés jusqu'ici, s'est re-positionnée une notion, celle d'expérimentation sociale. Son ambition est de matérialiser ce qu'est la demande sociale dans le champ de la communication.

Comme le rappelait le ministre de la Recherche et de la Technologie lors du lancement de la mission «Technologie, Diffusion de la Culture et Communication»:

Pour n'avoir pas fait précéder dans le passé la diffusion des produits nouveaux de recherches sur leur impact social et culturel de nombreux échecs ont été constatés, des prototypes n'ont pas franchi le seuil des laboratoires, et des expérimentations techniques n'ont pas été acceptées par le tissu social ${ }^{31}$.

Expérimenter l'acceptabilité socio-culturelle des nouvelles technologies, c'est adapter l'offre technologique à la demande du public, tenter d'échapper à la «logique de production économique», mettre au poste de commande un contrôle social et démocratique des outils technologiques, mais c'est aussi tenter d'éviter des rejets, des retards de socialisation des innovations par le marché.

Disons-le d'emblée. Si l'expérimentation sociale ne veut pas re-arraisonner une fois de plus les stratégies de commercialisation ou de création de besoins par le marketing, si elle ne veut pas en être réduite à construire des paravents sociaux pour des vitrines technologiques, si elle veut être crédible comme stratégie de changement social, elle doit s'inscrire dans le contexte des enjeux de la redéfinition de l'État. Car la question fondamentale réside bien là: de quels partenaires sociaux s'agit-il? Ce qui revient à se demander: comment germe une demande sociale? Comment émerget-elle? Comment se capte-t-elle? Sûrement pas à partir d'une commande des grandes institutions d'État. Sûrement pas à partir des besoins d'expansion du marché. Ou du moins pas exclusivement. C'est. là que le débat défini en termes public/privé dans le sens État/entreprise commerciale affiche ses limites. On ne peut le dépasser que si on s'interroge davantage sur un troisième pôle - un privé non commercial - que certains appellent déjà le tiers secteur tandis que d'autres préfêrent rester avec le terme de société civile (sans parfois se rendre compte des nouvelles logiques qui bouleversent l'État). Ce nouvel espace devrait refreiner, à la fois, la reproduction des «attitudes assistantielles» fomentées par l'État-providence, et l'emprise de la valorisation capitalistique.

Faute de la mise en place de nouveaux systèmes de médiation - pouvait-on lire dans une proposition qui se situait dans le droit fil des préoccupations émises lors du colloque - et en dépit de ce que la plupart des dirigeants socialistes actuels paraissent s'être dégagés des vieilles conceptions centralistes prétendant programmer le changement à partir des modèles idéologiques pré-établis, le risque demeure grand que les «raisons d'État», les «urgences» et les interties d'appareil nous conduisent à la même neutralisation bureaucratique de toutes les velléités de prise en charge des transformations sociales par les intéressés eux-mêmes et à un renforcement des attitudes collectives de dépendance à l'égard de l'État-provindence [...]. Le «tiers secteur» continuera d'être marginalisé et ne pourra guère jouer son rôle d'inducteur du changement, s'il ne parvient à instaurer un marché institutionnel, s'interposant

31. Lettre de J.P. Chevènement aux présidents de la mission «Technologie, diffusion de la culture et communication» (A. Mattelart et Y. Stourdzé) 15 mars 1982, dans Technologie, communication et culture, La Documentation française, 1982. 
entre le marché capitaliste, et les systèmes de contrôle étatiques et de sorte que puissent être mis à l'épreuve et confortés d'autres systèmes de valorisation des activités humaines, d'autres conceptions des finalités de la production sociale ${ }^{32}$.

Exprimé de façon plus précise à propos de l'innovation technologique et sociale dans un autre document, cela devient:

Les instances gouvernementales comme les grands partis ont à assumer l'usure classique du pouvoir et de la nouveauté. Cette usure, qui peut aller jusqu'à un tarissement complet des mesures sociales, ne sera évitée que si l'on favorise l'apparition et l'expression de forces de propositions ainsi que l'organisation d'espaces favorisant l'innovation et le partage des informations entre les différents groupes sociaux. Si une telle évolution n'était pas entreprise, on assisterait à une érosion progressive de la légitimité de l'État, érosion due spécialement à l'éloignement croissant des technostructures et du citoyen dans tous les grands secteurs: industrie, administration, syndicats, médias, etc.. La perte de légitimité ne sera enrayée que si l'on fait le pari de fonder le consensus social sur la reconnaissance et l'expression des multiplicités ${ }^{33}$.

On comprendra aisément que pour qui ne veut pas investir dans une vision mystifiante d'un «tiers-secteur» conçu comme valeur-refuge, c'est une analyse en profondeur de la complexité et des contradictions du tissu et des réseaux associatifs que convoquent ces réflexions. Un tissu associatif dont beaucoup se réclament mais sur le contenu duquel on n'est souvent pas d'accord. Car, à moins de s'abstraire de la longue histoire de la formation de la société civile française, force est de constater que dans ce tissu associatif coexistent aussi bien les vieilles associations (du type ligue de l'enseignement) au statut quasi parapublic, que les organisations nouvelles issues des luttes sociales hors usine des toutes dernières années ou encore celles qui, comme certains réseaux d'associations de parents, d'associations familiales ont dans les derniers temps pris en charge la critique des médias en dehors de tout circuit connu.

Sans aller plus avant dans une discussion qui devrait se poursuivre ailleurs, mais qui est rarement posée en ces termes, il est important de problématiser cette fameuse «expérimentation sociale». C'est autour de cette dernière que tourne dans la France d'aujourd'hui, à tort ou à raison, et malgré les connotations «d'expérience in vitro» qu'évoque inévitablement le terme même d'expérimentation, un des grands enjeux d'une redéfinition démocratique de la recherche en communication (entre autres). Elle oblige le chercheur à se situer dans sa façon de concevoir la science, dans sa manière d'imaginer son rapport avec le mouvement social.

Elle nous éloigne du cadre étroit de la seule revendication d'indépendance à l'égard de «toutes formes de pouvoir» qui se confond souvent - comme pour les journalistes - avec une croyance en l'indépendance des chercheurs à l'égard des rapports sociaux. Toutes croyances qui font que ce chercheur peut se permettre - parfois toute sa vie durant - de ne s'interroger ni sur les conditions de production de ses investigations, sur le pourquoi il est amené à choisir tel thème plutôt qu'un autre, ni sur les retombées de ce qu'il fait. En un mot, à quoi il sert et qui l'utilise. De ce point de vue, la simple confrontation de la trajectoire des sciences de la communication en France avec cette notion de «demande sociale» montrerait combien la grande majorité des recherches n'ont fait que répondre à la dynamique contraignante des demandes du pouvoir.

Que l'on accepte les concepts de demande sociale et d'expérimentation sociale, que l'on préfère substituer au dernier celui «d'intervention sociale», il est clair que l'on se trouve devant des enjeux dont l'issue incertaine sera, ou bien la possibilité enfin offerte, à travers un nouveau schéma de participation et de captation des innovations sociales et technologiques d'une réelle plus-value démocratique dans une société où la technologie de communications serait perçue et reçue comme partie intégrante des rapports sociaux et l'affaire de tous, ou bien la création d'un corps d'experts en ingénierie sociale, c'est-à-dire tout le contraire de ce que devrait être la science sociale, une contre-expertise, une subversion. L'enjeu est de taille car il est difficile de le situer hors d'issues plus générales à savoir la redistribution du pouvoir entre les différentes classes et groupes sociaux qui constituent la société française. Car c'est dans ce contexte de sortie politique de la crise que s'affirme l'aspiration de plus en plus hégémonique des couches professsionnelles et techniciennes (certains parleront de nouvelle petite bourgeoisie) à gérer la société.

32. F. Guattari, doc. cit.

33. M.J. Carrieu-Costa et M. Callon, Projet d' un centre de recherche et de formation sur les innovations technologiques et sociales, mars 1982, ronéo. 


\section{RENDRE À L'EXPÉRIMENTATION SOCIALE SES CONTRADICTIONS}

Les quelques notes qui suivent sont à verser au dossier des discussions déjà nombreuse ${ }^{34}$ sur la nécessité de «conflictualiser» la notion de demande sociale et d'autres qui peuvent s'inscrire dans sa mouvance.

1. Tout projet politique qui ne se contente plus de proclamer les vertus orthopédiques intrinsèques à ces technologies mais au contraire accepte de poser le rapport technologie/démocratie, technologie/demande sociale est, dans le cadre de la politique actuelle de sortie de crise (voir supra) choisie par le gouvernement socialiste, forcément habité par des tensions. Il tente de réconcilier deux logiques, deux nécessités, apparemment irréconciliables. Il s'agit d'une part, de permettre à des industries de conquérir un marché, c'est-à-dire en fait, multiplier les interfaces homme-machine, impulser l'électronisation du mode de communication entre les individus; d'autre part, il s'agit d'accepter que l'expansion de ce marché se voie éventuellement freiné par une demande sociale, à savoir l'expression de besoins qui peuvent bien ne pas se satisfaire de ce seul mode de communication sur support électronique. Bref, le mot d'ordre industriel - ou pour reprendre les termes du président de la République au Sommet de Versailles «le soutien de la demande afin de favoriser le développement des marchés pour les nouveaux biens et services de consommation incorporant les progrès technologiques ${ }^{35} \gg$ - risque de contrevenir à l'impératif de la démocratie.

C'est là qu'une certaine conception de l'expérimentation sociale montre rapidement son véritable motif et ses possibles impasses. Car toutes les logiques sociales n'admettent pas nécessairement une issue technologique. L'entrée dans l'expérimentation sociale par la voie technologique conditionne déjà un découpage de la réalitée ${ }^{36}$. On ne peut, en effet, se dérober devant une question liminaire: les gens ont-ils réellement besoin de cette technologie?

Appréhender la demande sociale sous cette perspective, implique d'aborder l'évaluation des expériences de communication réalisées sur support technique (vidéo, radios, réseaux télématiques) d'une façon nouvelle. Car les retombées secondes de ces expériences ne sont pas nécessairement des retombées technologiques, (ou du moins des retombées dans le mode technologique que l'on a privilégié au départ): de l'évolution des télévisions communautaires vers les radios communautaires au Québec, du glissement de la vidéo-gazette vers des expériences originales de pratiques pédagogiques

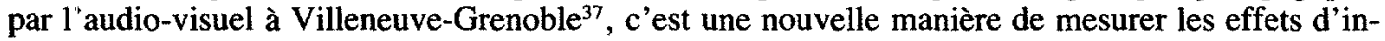
terventions sociales que l'on a tendance - une fois échouées - à jeter aux oubliettes au nom d'une théorie de la fatale récupération des innovations sociales (dites marginales ou alternatives) par le système établi. Point n'est besoin d'ajouter que ce schéma d'interprétation qui n'envisage les innovations sociales que dans leur rôle transitoire ou de régulation vient souvent à point nommé pour les divers pouvoirs locaux ou nationaux. Car à force de ne voir ces expériences que comme des échecs - sans même se demander de quel échec il s'agit et de quels acteurs sociaux cela a été l'échec - , se réalimente la cause de tous les ennemis d'une décentralisation démocratique des réseaux non seulement de distribution mais de production de la communication et de la culture. Dans un tel scénario reposant sur le couple innovation-intégration, innovation-récupération (récupération

34. Voir par exemple le dossier «Pour une autre télévision», NON! (repères sur le socialisme) janvier-février 1982 (avec des contributions de A. Spire et D. Goldschmidt); Y. de La Haye et B. Miège, «Les socialistes français aux prises avec la question des media», Raison présente, Paris, premier trimestre 1982; P. Flichy, «Pour une politique de l'innovation sociale en audiovisuel» Le monde diplomatique, mai 1982.

35. Président F. Mitterand, Sommet de Versailles, doc. cit.

36. Voir à titre d'exemple C. Collin, "Villeneuve: de la vidéogazette au centre de ressources», Sonovision, juilletaoût 1978. Une mention spéciale mériterait ici l'Italie et son expérience de radios et télévisions libres. Non pas en tant qu'elle-même, mais en tant qu'elle sert de support à de nombreux mythes, agités également par les ennemis de la démocratisation de la communication. Combien de fois n'a-t-on pas vu ici en France les partisans d'une démocratie restreinte dans les media fermer toute discussion en invoquant la situation italienne.

37. Avec justesse le groupe de travail sur l'expérimentation sociale de la commission Moinot notait en juillet-août 1981 qu'il était possible d'adapter deux démarches expérimentales différentes:

«La première consiste à partir du système technologique que l'on souhaite tester et à observer la manière dont un milieı l'adopte, le rejette ou le transforme. S'il est nécessaire à un organisme se préoccupant de l'avenir de la communication de se tenir informé de ces expériences, voire d'y être associé de façon à en pénétrer les mécanismes, on peut se demander s'il n'est pas possible de retourner la question et d'envisager des formes d'expérimentation différentes: au lieu de partir de la technique et de s'interroger sur ses usages, partir de pratiques sociales innovantes et rechercher quelle réponse technique peut être apportée aux besoins en matière de communication. Les nouveaux moyens pourront certes être utilisés mais seulement dans la mesure où il apparaîtra qu'ils répondent mieux que les media déjà connus et éventuellement, sous forme d'une combinaison avec ces media, aux besoins exprimés par les utilisateurs. De telles expérimentations pourraient être mises en cuvre en utilisant les réseaux existants sans attendre l'installation de nouveaux réseaux». 
par la structure administrative ou par la structure classique marchande), il n'y a pas d'espace pour un débat sur les possibilités réelles de redéfinition démocratique de la société civile dont nous parlions plus haut. Consolons-nous cette société a fort heureusement des retours de flamme que ne prévoient pas ces conceptions «dualistes» et manipulatoires du pouvoir!

2. Quelques remarques finales sur le risque «d'autonomisation» du débat sur les nouvelles technologies de communication dans la France d'aujourd'hui. Et donc sur le risque de considérer superflue toute discussion sur ce qu'est la démocratie. La fascination pour la technologie - qui prend appui sur un néo-positivisme ambiant — fait des ravages. On assiste même à une recrudescence des dérives technicistes. L'univers de la «communication» devient un espace privilégié de neutralisation du politique, un univers euphémisé d'où seraient absents les conflits d'intérêts, les rapports de forces, les clivages et les pesanteurs sociologiques. Aux termes de son étude sur l'introduction de l'audiovisuel en bibliothèque, J.C. Passeron rappelait avec justesse:

Autre prudence, pour ne pas retomber sans cesse de très haut dans la déception: y regarder à deux fois avant de tout miser sur le dernier avatar de l'optimisme diffusionniste. On voit, à chaque époque, le dernier-né des progrès technologiques redonner vie à l'espoir scientiste que la multiplication des biens culturels ou leur accessibilité accrue vont opérer comme par enchantement le «salut culturel» des masses. Aucune innovation technologique n'a jamais eu raison, par la seule grâce du médium, des inégalités culturelles produites et reproduites par le jeu bien rôdé des structures et des hiérarchies sociales: les caractéristiques techniques d'un moyen de communication ne pré-déterminent jamais ses éffets sociaux au point d'exclure tout effet tenant aux rapports sociaux qui président à l'utilisation de cette technique ${ }^{38}$.

a/Tout se passe dans certains secteurs comme si le fait d'être au pouvoir, de ne plus appartenir à l'éternelle opposition, avait signifié la conquête d'une bonne conscience technologique, mieux, une légitimité technologique.

Les raisons de cette fascination plongent leurs racines dans une histoire à la fois ancienne et récente. Histoire ancienne: les forces de gauche $n$ 'ont, en fait, jamais réglé leurs comptes avec la conception instrumentaliste de la technologie et ont revalidé pour l'essentiel la croyance en ce que les questions politiques que l'on pouvait adresser à la technologie se résolvaient au niveau du bon ou mauvais usage des outils offerts par le progrès technique. Dans une telle conception, prend difficilement place par exemple une interrogation critique sur le rapport qu'entretient la technique avec la reproduction de la division sociale du travail, la ségrégation entre les propriétaires du savoir/information et les autres. Or, cette interrogation serait particulièrement stratégique en ce moment où la société française doit répondre à un défi technologique intimement lié au défi politique de restructuration des rapports de force entre les groupes et les classes, en ce moment où l'information, la possibilité de la produire et de contrôler son mode d'emploi, devient de plus en plus l'axe central de réorganisation de tout type de pouvoir et de l'approfondissement de la division sociale.

Histoire récente: pas moyen en effet, — c'est une banalité de le dire-, d'expliquer ce refuge dans le technologique sans le relier à un processus global: crise de légitimité des grandes organisations traditionnelles qui ont infléchi le cours des logiques de domination depuis le début de l'organisation de la classe ouvrière, crise des grands idéaux sociaux (et de l'idée même d'égalité), toutes composantes d'une crise profonde du «politique», des formes de le pratiquer et de sa définition. Crise qu'une victoire électorale peut seulement déplacer, mais qui revient avec d'autant plus de forces que certains avaient cru la conjurer une bonne fois pour toutes les grands soirs de la Bastille.

b/Une technologie chasse l'autre. La loi de l'obsolescence est la loi d'un certain progrès technologique. Avec la fascination technologique, c'est l'histoire de la longue accumulation des pratiques de résistances antérieures, dans le champ de la communication, qui risque de disparaître. L'histoire de cette accumulation est balayée par l'artefact - dernier arrivé car la logique du marché est de fonctionner en temps réel. Le procès d'atomisation opère à un double niveau: celui du temps (on recommence à zéro à chaque nouvelle technologie): celui de l'espace (on perd la mémoire d'un possible réseau de solidarité à partir de pratiques sociales multiples et complémentaires).

En ne reprenant pas les acquis et les échecs des expériences antérieures, en ne réévaluant pas ces dernières, en ne les croisant pas entre elles, c'est le refus de construction d'une théorie de la communication qui s'affirme. C'est aussi le refus de tenir compte des apports multiples d'autres

38. J.C. Passeron, «Images en bibliothèque, images de bibliothèques», Paris, Documents du Gides, janvier 1982, pp. 46-47. 
recherches, d'autres disciplines, d'autres expériences qui en d'autres champs se développent. De là par exemple, l'ingénuité de certains communicologues qui font fi soit des recherches sur la reproduction sociale à l'école, soit de celles qui ont approché les politiques de démocratisation à travers l'action et l'animation culturelles, soit de celles, souvent pionnières, qui se sont penchées sur l'aménagement de l'espace urbain, enfin de celles qui à partir de l'anthropologie, la littérature, l'histoire ont tentê de cerner le champ des cultures populaires d'hier et d'aujourd'hui.

c/ C'est dans ce contexte global que travaille une autre fascination, qui a pour objet le local. Las des grands appareils broyeurs de spécificité et d'identité particulières, le local est apparu, à certains secteurs, depuis déjà quelques années comme forcément synonyme de retrouvailles avec une proximité et une convivialité perdues. Aujourd'hui que le mot d'ordre de la décentralisation s'est installé officiellement, le local s'est affirmé comme un lieu important de redéfinition de l'État où $\mathrm{plus}$ que jamais s'affrontent des projets violemment contradictoires ${ }^{39}$.

À n'en pas douter, l'issue de la décentralisation se joue entre l'approfondissement du processus d'atomisation, d'éparpillement face à un pouvoir télécentré et l'instauration de nouvelles formes et réseaux de solidarité. Tout comme se fait sentir de plus en plus les besoins d'un débat sur l'articulation national/transnational pour juger d'une politique d'indépendance et de reconstruction de l'identité nationale, surgit avec force l'urgence de s'interroger sur l'articulation entre le local et le national, le local et le global, pour juger d'une politique de redistribution du pouvoir. C'est là que la notion de «réseaux» montre son utilité lorsqu'on cherche à échapper aux enfermements localistes ${ }^{40}$.

39. Sur les enjeux du «local» voir A. Mattelart et J.M. Piemme, Télévision: enjeux sans frontières, Presses universitaires de Grenoble, 1980; J.P. Garnier, «De la crise d'une gestion à la gestion d'une crise ou le charme discret de la décentralisation», Cahiers secteur public, Paris, janvier 1982.

40. C'est là qu'une réflexion sur des expériences comme celle du réseau ANTELIM qui a patiemment tissé des liens ladiophoniques entre les familles des marins permettrait de pondérer cet enthousiasme démesuré pour les radios locales, impulsées officiellement par le plan de décentralisation du monopole public de radio-télévision. 